EDITORIAL

\title{
Cost-Effectiveness of Pharmacological Treatment in Vascular Dementia
}

Can. J. Neurol. Sci. 2009; 36: 673-674

Dementia is a common and costly disease. Data from the Canadian Study of Health and Aging (CSHA) suggest that more than $35 \%$ of individuals aged 85 and older are affected by dementia $^{1}$. The annual cost of dementia in the United Kingdom is estimated to be higher than the combined costs associated with heart disease, cancer, and stroke ${ }^{2}$. Most cases of dementia are thought to be due to Alzheimer's Disease with (so called "mixed dementia") or without cerebrovascular contribution ${ }^{3,4}$. Vascular dementia $(\mathrm{VaD})$ was traditionally considered to be the second most common cause of dementia ${ }^{1}$. Recent epidemiological, neuroimaging and clinico-pathological studies have significantly improved our understanding of the cerebrovascular contribution to dementia and have shed some doubt on the traditional conception of $\mathrm{VaD}^{5,6}$. Multi-infarct dementia (characterized by macrovascular lesions), which was once considered the prototype of $\mathrm{VaD}$, is now thought to be just one aspect of a far more diverse category. The constant sophistication of neuroimaging techniques shows a significant contribution of microvascular disease to $\mathrm{VaD}$. The traditional view of dementia as a disease primarily affecting memory and other cortical cognitive functions has also limited clinical characterization of $\mathrm{VaD}$ in which lesions (especially small vessel disease) typically affect sub-cortical structures and spare memory in early stages ${ }^{7}$. These factors have led to a reevaluation of $\mathrm{VaD}$ and the introduction of the concept of Vascular Cognitive Impairment $(\mathrm{VCI})^{8,9}$. According to this novel concept, the etiology of the clinical deficit would consider the diverse types of vascular insults. The cognitive evaluation would take into account the particular neuropsychological manifestations caused by these lesions. In VCI, the definition of impairment moves away from the "all or nothing" view of traditional dementia and allows for the inclusion of individuals with less severe deficits ("brain at risk" and "cognitive impairment without dementia" stages), underscoring the need and relevance for prevention. Harmonization standards for VCI have been proposed and will need to be considered in future studies of dementia with a cerebrovascular contribution ${ }^{10}$.

Studies of $\mathrm{VaD}$ show the presence of a cholinergic deficit suggesting that cholinesterase inhibitors (ChEI), usually used in Alzheimer's Disease, may be useful in this condition ${ }^{11,12}$. Three ChEI's are available on the Canadian market: donepezil, rivastigmine and galantamine. Several clinical trials of ChEI's in $\mathrm{VaD}$ have been conducted. A metanalysis including eight randomized controlled trials with ChEI's and memantine (a noncompetitive N-Methyl-D-Aspartic acid antagonist) concluded that they provided small but significant benefits in cognition in patients with mild to moderate $\mathrm{VaD}^{13}$. The use of these drugs, however, was associated with more adverse events and dropouts than placebo. Such studies have led the most recent Canadian Consensus Conference of Diagnosis and Treatment of Dementia to conclude: "There is fair evidence of benefit of small magnitude for donepezil in cognitive and global outcomes, with less robust benefits on functional measures. Donepezil can be considered a treatment option for vascular dementia (Level I, grade B recommendation)" 14 . The specific recommendation of Donepezil in this context was based on the number and quality of published studies. None of the ChEI's nor memantine are currently approved for use in $\mathrm{VaD}$ in Canada.

The paper by Wong et al published in this issue of the Journal sheds new light on the clinical decision to use ChEI's in $\mathrm{VaD}^{15}$. They conduct the first cost-effectiveness evaluation study of ChEI's and memantine in VaD. In their model, the authors used efficacy and adverse event data from the above-mentioned metanalysis. In their determination of incremental cost of ChEI, they considered cost of the individual medications and of physician services associated with follow-up and management of their side-effects. The cost was reported in 2008 Canadian dollars, and expressed per unit decrease (or improvement) on the Alzheimer's Disease Assessment Scale - cognitive subscale (ADAS-Cog). Obviously, the incremental costs for all medications were higher than standard care (no pharmacological treatment). Donepezil $10 \mathrm{mg}$ was associated with the lowest incremental cost-effectiveness ratio, estimated at $\$ 400.64$ per unit improvement on the ADAS-Cog. In their conclusions, authors of the study recommend that donepezil should be considered if ChEI's met regulatory approval for $\mathrm{VaD}$. $\mathrm{T} \mathrm{h}$ i s innovative and rigorously conducted study addresses an issue that is neglected in clinical trials with antidementia medications. Therefore, lack of cost-effectiveness studies in dementia (and $\mathrm{VaD}$ in particular) is often an argument used by skeptics to justify lack of pharmacological intervention. Unfortunately, this study suffers from limitations of the clinical trials used in the cost-effectiveness model. Improvement on a cognitive scale such as the ADAS-Cog cannot readily be translated into real-life benefit. On the other hand, several very costly aspects of dementia care could not be analyzed in this study as they were not evaluated in the clinical trials. These include: delaying of functional decline or emergence of behavioral complications, nursing home placement, caregiver burden, indirect costs such as absenteeism from work, etc. Therefore, potential cost-savings with these medications could not be determined. Also, in the analysis of cost, authors assume that "minor" adverse events are managed through a visit to the physician and add this to the general calculation of cost. Practically, clinicians would probably agree that most of these side-effects can be managed over the phone by a physician or a trained nurse. Hence, the costincrement calculated in this study represents at best an overestimation of the cost associated with use of these medications. The specific recommendation to use donepezil in this context relies on evidence-based data. However, clinical experience 
with ChEI's suggests that most of their benefits are probably class effects.

Clinicians need to keep in mind that the role of ChEI's in dementia is symptomatic. Expectations need to be adapted accordingly. Nevertheless, the (modest) benefits provided by these medications are constant and reproducible across clinical trials. The results of this study will reassure the physicians who already use $\mathrm{ChEI}$ in $\mathrm{VaD}$. It may also encourage those who don't to be more proactive and use these medications in patients with $\mathrm{VaD}$. As expensive disease-modifying treatments are being developed and explored for dementia, one hopes that costeffectiveness analyses such as the one by Wong et al, will become integral parts of clinical trials to better help patients, clinicians and third party payers in their decisions about pharmacological treatment ${ }^{15}$.

\section{DisClosures}

Fadi Massoud has received speaker's honoraria, consultant fees, and research support from Pfizer, Janssen Ortho Inc., Novartis, and Lundbeck.

\section{REFERENCES}

1. Canadian Study of Health and Aging. Study methods and prevalence of dementia. CMAJ. 1994;150:899-913.

2. Burns A, Lliffe S. Dementia. BMJ. 2009;338:405-9.

3. Feldman H, Levy AR, Hsiung GY, Peters KR, Donald A, Black SE, et al. A Canadian cohort study of cognitive impairment and related dementias (ACCORD): study methods and baseline results. Neuroepidemiology. 2003;22:265-74.

4. Chertkow H. Diagnosis and treatment of dementia: introduction. CMAJ. 2009;178:316-21.

5. Lopez OL, Kuller LH, Becker JT, Jagust WJ, DeKosky ST, Fitzpatrick A, et al. Classification of vascular dementia in the Cardiovascular Health Study Cognition Study. Neurology. 2005; 64:1539-47.

6. Bruandet A, Richard F, Bombois S, Maurage CA, Deramecourt V, Lebert F, et al. Alzheimer disease with cerebrovascular disease and vascular dementia: clinical features and course compared with Alzheimer disease. J Neurol Neurosurg Psychiatry. 2009; 80:133-9.

7. Hachinski V. Vascular dementia: a radical redefinition. Dementia. 1994;5:130-2.

8. Bowler JV, Hachinski V. Vascular cognitive impairment: a new approach to vascular dementia. Baillieres Clin Neurol. 1995;4: 357-76.

9. O'Brien JT, Erkinjuntti T, Reisberg B, Roman G, Sawada T, Pantoni L, et al. Vascular cognitive impairment. Lancet Neurol. 2003;2: 89-98.

10. Hachinski V, Iadecola C, Petersen RC, Breteler MM, Nyenhuis DL, Black SE, et al. National Institute of Neurological Disorders and Stroke-Canadian Stroke Network vascular cognitive impairment harmonization standards. Stroke. 2006;37:2220-41.

Fadi Massoud Montreal, Quebec

11. Grantham C, Geerts $H$. The rationale behind cholinergic drug treatment for dementia related to cerebrovascular disease. J Neurol Sci. 2002;203-204:131-6.

12. Roman G, Kalaria R. Vascular determinants of cholinergic deficits in Alzheimer disease and vascular dementia. Neurobiol Aging. 2006;27:1769-85.

13. Kavirajan H, Schneider LS. Efficacy and adverse effects of cholinesterase inhibitors and memantine in vascular dementia: a meta-analysis of randomised controlled trials. Lancet Neurol. 2007;6:782-92.

14. Bocti C, Black S, Frank C. Management of dementia with a cerebrovascular component. Alzheimers Dement. 2007;3: 398-403.

15. Wong CL, Bansback N, Lee PE, Anis AH. Cost-effectiveness: cholinesterase inhibitors and memantine in vascular dementia. Can J Neurol Sci. 2009;36:735-9. 